\section{Estudo domiciliar da população idosa de Fortaleza/CE: aspectos metodológicos e características sócio-demográficas*}

\section{Household survey of the Elderly Population of Fortaleza/CE: methodological issues and socio- demographic characteristics}

Tarciana Nobre de Menezes ${ }^{1}$

Francisco José Moreira Lopes ${ }^{2}$

Maria de Fátima Nunes Marucci ${ }^{3}$

'Núcleo de Estudos e Pesquisas Epidemiológicas - NEPE, Universidade Estadual da Paraíba/UEPB

${ }^{2}$ Unidade Estadual do IBGE no Ceará

${ }^{3}$ Departamento de Nutrição da Faculdade de Saúde Pública, Universidade de São Paulo/USP

"Trabalho elaborado a partir de dados do estudo "Estado nutricional, valor energético da alimentação dos idosos de Fortaleza/CE e sua associação com sexo, grupo etário e nível sócio-econômico". Apoio financeiro: Secretaria de Saúde do Estado do Ceará (SESA) em convênio com o Centro de Treinamento e Desenvolvimento (CETREDE) (Processo n 02429195-1).

Correspondência: Tarciana Nobre de Menezes. Avenida das Baraúnas, 351. Campus I, Prédio dos Mestrados. $2^{\circ}$ and - Bodocongó, Campina Grande - PB, CEP: 58109-753. E-mail: tnmenezes@yahoo.com.br

\section{Resumo}

Objetivo: Descrever a metodologia do estudo e identificar as características sóciodemográficas dos idosos residentes na cidade de Fortaleza/CE. Metodologia: Este estudo caracteriza-se por ser epidemiológico, do tipo transversal e de base domiciliar. Foram selecionados 385 domicílios com base em técnicas de processos probabilísticos com estratificação dos bairros e setores censitários de Fortaleza. As variáveis utilizadas neste estudo foram: sexo, grupo etário (60-69, 70-79 e 80 anos ou mais), cor, escolaridade, estado civil, número de residentes no domicílio. Resultados: Foram entrevistados 483 idosos (327 mulheres e 156 homens). Os idosos pardos representaram $55,1 \%$ da amostra. A maioria dos idosos $(61,7 \%)$ era analfabeta ou cursou o primeiro grau incompleto. A maior parte era casada $(48,5 \%)$; no entanto, entre as mulheres, houve elevada proporção de viúvas (38,2\%). Em $68,7 \%$ dos domicílios visitados, havia de 2 a 5 residentes. Conclusão: Os resultados mostram a heterogeneidade da população idosa. Diante disso, observa-se a importância do planejamento de políticas públicas direcionadas às necessidades específicas da população idosa, além de outros estudos que possibilitem ampliar o conhecimento a respeito dessa população.

Palavras-chave: Idoso. Estudo epidemiológico. Pesquisa domiciliar. Características sócio-demográficas. 


\section{Abstract}

Objective: To describe the study methodology and to identify the socio-demographic characteristics of the elderly in Fortaleza/CE. Method: Epidemiological, cross-sectional household-based study. A total of 385 households were selected through probabilistic sampling techniques stratified by the neighborhoods and census sectors of Fortaleza. The variables analyzed were: gender, age group (60-69, 7079 and 80 years or more), skin color, years of schooling, marital status and number of residents in the household. Results: 483 elderly people were interviewed (327 women, $126 \mathrm{men}$ ). Elderly mulattos represented $55.1 \%$ of the sample. Most of the elderly $(61.7 \%)$ were illiterate or had a very low educational level. A large number of the elderly was married (48.5\%); however, there was a higher proportion of widows $(38.2 \%)$ among women. $68.7 \%$ of the selected households had between 2 and 5 residents. Conclusion: Results indicate the heterogeneity of the elderly population. These results suggest the importance of public action to meet the needs of the elderly population. In addition, other studies should be performed, in order to expand the knowledge on this population.

Keywords: Elderly. Epidemiological study. Household survey. Socio-demographic characteristics.

\section{Introdução}

O envelhecimento populacional é um fenômeno que vem acorrendo tanto nos países desenvolvidos, onde já se encontra em fase de estabilização, quanto nos países em desenvolvimento, onde esse processo encontra-se mais acelerado, como é o caso do Brasil. Alguns autores afirmam que esse fenômeno deve-se principalmente ao aumento da expectativa de vida e à queda dos coeficientes de mortalidade e fecundidade ${ }^{1,2}$. De acordo com Carvalho e Garcia $^{3}$, no Brasil, o envelhecimento populacional é devido à rapidez e manutenção da queda dos índices de fecundidade. Os autores afirmam, ainda, que haverá aceleração do processo de envelhecimento, caso haja declínio substancial da mortalidade nas idades avançadas.

Há projeção de que, em 2080, no mundo desenvolvido, $20 \%$ da população terá mais de 65 anos $^{4}$, enquanto estima-se que, em 2025, a população idosa no Brasil será de 30 milhões de pessoas, o que corresponderá a $15 \%$ da população ${ }^{5}$.

A cidade de Fortaleza possui 2.141.402 habitantes e, destes, 160.231 (7,5\%) são indivíduos com 60 anos ou mais e assim como acontece no Brasil, Fortaleza tem experimentado aumento substancial na população idosa ${ }^{6}$. De acordo com dados dos Censos Demográficos realizados nos anos de 1980 e 2000, constata-se que o crescimento da população idosa de Fortaleza em 20 anos foi expressivo, em relação à população total, visto que, enquanto houve aumento de $63 \%$ na população total, houve aumento de $130 \%$ na população idosa (60 anos e mais) ${ }^{7,8}$.

Considerando os aspectos apresentados e a importância de pesquisas com idosos, este estudo teve como objetivo identificar as características sócio-demográficas dos idosos residentes na cidade de Fortaleza/CE.

\section{Metodologia}

Este trabalho é parte de um estudo 
maior intitulado "Estado nutricional, valor energético da alimentação dos idosos de Fortaleza/CE e sua associação com sexo, grupo etário e nível sócio-econômico", que é composto, ainda, por informações antropométricas, de consumo alimentar e do nível sócio-econômico. Consiste em um estudo populacional, de base domiciliar, do tipo transversal, com coleta de dados primários, constituído por indivíduos com 60 anos ou mais, de ambos os sexos, residentes habituais em domicílios particulares da cidade de Fortaleza/CE, os quais foram selecionados para o estudo.

Foram excluídos do estudo idosos que apresentassem alguma das seguintes situações: paciente em fase terminal de doença, com debilidade clínica grave, ausência de informante adequado, caso houvesse necessidade, idoso que estivesse ausente de Fortaleza por mais tempo que a pesquisa de campo naquele setor.

\section{Plano de amostragem}

A amostra para o estudo foi calculada em função do número de domicílios de Fortaleza, cujo dimensionamento e seleção foram sistematizados com assessoramento técnico da unidade estadual do Instituto Brasileiro de Geografia e Estatística (IBGE).

O plano de amostragem foi elaborado com base em técnicas de processos probabilísticos com estratificação dos bairros e setores censitários ${ }^{*}$ de Fortaleza, para garantir a representatividade da área territorial em que a pesquisa foi realizada, o que permite que, nos domicílios selecionados as informações obtidas junto aos idosos possam produzir resultados válidos para o conjunto do qual a amostra foi retirada.

\section{Seleção da amostra}

A seleção da amostra foi realizada considerando a divisão de Fortaleza, de acordo com o IBGE, em 9 regiões administrati- vas (Centro, Mucuripe, Cidade dos Funcionários, Messejana, Prefeito José Walter, Parangaba, Conjunto Ceará, Antônio Bezerra e Barra do Ceará). De cada região administrativa foram sorteados 2 setores censitários, totalizando os 18 setores que constituíram a amostra deste estudo (Tabela 1). Para que a amostra fosse representativa do universo, as técnicas amostrais foram planejadas em múltiplas etapas, cuja seleção foi desenvolvida em estágios sucessivos, a seguir especificados.

Primeiro estágio - Seleção aleatória dos bairros em cada região administrativa, bem como definição do número de domicílios a serem visitados em cada bairro. Fixou-se em dois o número de bairros por região.

Segundo estágio - Seleção aleatória dos setores censitários em cada bairro sorteado, fixando-se um setor por bairro, mediante mapeamento do IBGE.

Terceiro estágio - Identificação do domicílio a ser visitado, de forma sistemática.

\section{Seleção do domicílio}

A identificação do primeiro domicílio ocorreu aleatoriamente, considerando como ponto de partida a esquina inicial do setor, em sentido horário. O número de domicílios a serem percorridos até chegar ao próximo a ser visitado foi definido a partir da razão entre o número total de domicílios no bairro e o número de domicílios determinados para serem visitados naquele bairro, gerando-se, assim o número 12 (Tabela 1). Dessa forma, a cada domicílio selecionado, foram saltados onze domicílios. O $12^{\circ}$ domicílio foi o visitado, e assim sucessivamente, a fim de obter-se melhor distribuição e garantia de que todo o setor seria visitado.

Foi considerado domicílio o local utilizado como habitação de uma ou mais pessoas, ou que estivesse sendo utilizado para

'Setor censitário é definido, pelo IBGE, como sendo uma área territorial de pesquisa formada de área contínua, situada em um único quadro urbano ou rural, com dimensão e número de domicílios ou de estabelecimentos que permitam o levantamento de informações, com limites bem determinados, tendo em média 300 domicílios em sua área. 
Tabela 1 - Regiões administrativas, bairros, número de domicílios selecionados por bairro e número de idosos entrevistados da cidade de Fortaleza/CE.

Table 1 - Administrative regions, neighborhoods, number of households selected per neighborhood and number of elderly individuals interviewed in the city of Fortaleza/CE.

\begin{tabular}{lcccc}
\hline Região administrativa & Bairro & $\begin{array}{c}\text { №total de } \\
\text { domicílios }\end{array}$ & $\begin{array}{c}\text { № de domicílios } \\
\text { selecionados }\end{array}$ & $\begin{array}{c}\text { № de idosos } \\
\text { entrevistados }\end{array}$ \\
\hline Centro & Dionísio Torres & 163 & 13 & 16 \\
Centro & Parquelândia & 288 & 24 & 27 \\
Mucuripe & Meireles & 316 & 26 & 33 \\
Mucuripe & Papicu & 300 & 25 & 33 \\
Cid. Funcionários & Cid. Funcionários & 319 & 26 & 34 \\
Cid. Funcionários & Edson Queiroz & 425 & 35 & 45 \\
Messejana & Lagoa Redonda & 194 & 16 & 20 \\
Messejana & Messejana & 212 & 17 & 23 \\
Pref. José Walter & Pref. José Walter & 185 & 15 & 34 \\
Pref. José Walter & Maraponga & 317 & 26 & 28 \\
Parangaba & Montese & 242 & 20 & 14 \\
Parangaba & Alto da Balança & 175 & 14 & 22 \\
Conj. Ceará & Canindezinho & 214 & 18 & 28 \\
Conj. Ceará & Granja Lisboa & 312 & 26 & 29 \\
Ant. Bezerra & Dom Lustosa & 271 & 22 & 30 \\
Ant. Bezerra & Quintino Cunha & 261 & 22 & 19 \\
Barra do Ceará & Barra do Ceará & 231 & 19 & 25 \\
Barra do Ceará & Cristo Redentor & 254 & 21 & \\
\hline
\end{tabular}

este fim. Para ser considerado como unidade domiciliar para a pesquisa, o domicílio precisava satisfazer simultaneamente a duas condições: separação e independência ${ }^{9}$.

A condição de separação do domicílio deveria satisfazer simultaneamente às seguintes condições: ser limitado por paredes, muros, cercas; ser coberto por um teto; permitir que a pessoa ou o grupo de pessoas que nele habitasse se isolasse das demais; e que a pessoa ou o grupo de pessoas que nele habitasse arcasse com parte ou com todas as suas despesas de alimentação e moradia.

A condição de independência se caracterizou pelo acesso direto ao domicílio, permitindo que seus moradores pudessem entrar ou sair sem passar por locais de habitação de outras pessoas.

No caso de edificações com várias unidades, primeiramente, o entrevistador verificava se o edifício era residencial, não residencial ou misto. Seguia listando do andar mais alto para o mais baixo. Não fo- ram descartadas unidades localizadas em coberturas, subsolos e locais destinados a alojamento de porteiros, zeladores e faxineiros. As unidades de um mesmo pavimento foram listadas de acordo com a seqüência encontrada (numérica, alfabética). Unidades constituídas por estabelecimentos coletivos como hospitais, clínicas, escolas e igrejas (desde que não morasse alguém), clubes, quartéis e similares, mesmo que destinados aos idosos, foram excluídos da seleção aleatória, por conseguinte, do estudo.

\section{Seleção do idoso}

Caso não residisse idoso no domicílio selecionado ou o mesmo não aceitasse (recusa) ou não pudesse (perda) participar do estudo, outro idoso foi procurado no domicílio seguinte.

Quando residia idoso no domicílio, mas o mesmo estava ausente, eram realizadas até mais duas visitas de retorno. A contagem dos domicílios para a próxima 
entrevista continuava considerando este domicílio como parte da amostra. Caso o idoso não fosse encontrado, após as duas visitas de retorno, o mesmo era considerado como perda e substituído por outro (s) idoso (s) residente em um domicílio no mesmo setor, seguindo a contagem pelo último domicílio visitado.

Caso acontecesse de no domicílio selecionado, residir um idoso, que estivesse temporariamente ausente da residência habitual no momento da entrevista, no entanto estivesse em Fortaleza, o mesmo era visitado no local de estada atual. Quando no domicílio residia mais de um idoso, todos foram convidados a participar do estudo.

Foi feito o possível para que todos os idosos participassem do estudo, mesmo que pudessem apresentar alguma limitação ou problema de saúde. Quando isso não foi possível, recorria-se a um informante, que poderia ser auxiliar ou substituto. No caso de o entrevistado apresentar condições de fornecer a maior parte das informações, porém necessitasse da ajuda de outra pessoa para responder a determinadas perguntas, a pessoa, no caso o informante auxiliar, ajudava o idoso. Quando o idoso apresentava alguma limitação ou problema de saúde grave que o impedisse de fornecer todas ou a maior parte das informações, era requisitado um informante substituto.

O fator principal a ser considerado para a escolha do informante foi que o mesmo fosse integrante do domicílio e que estivesse em contato regular e estreito com o entrevistado, de forma que soubesse responder a respeito do que trata a pesquisa: data de nascimento, escolaridade, posses, alimentação. Nesse caso, as pessoas mais indicadas foram: o cônjuge, os filhos, as noras ou outros familiares ou amigos que dessem apoio diário ou freqüente ao entrevistado.

\section{Trabalho de campo}

O trabalho de campo foi desenvolvido com três equipes de dois entrevistadores cada uma. As equipes que foram a campo para realizar a coleta dos dados eram constituídas por alunos do curso de Nutrição da Universidade Estadual do Ceará (UECE), os quais foram treinados pela autora desta pesquisa, a qual possui experiência em pesquisa com idosos, uma vez que realizou na cidade de Fortaleza estudo com 305 idosos institucionalizados ${ }^{10}$.

Os entrevistadores foram a campo em dupla, devidamente identificados, munidos de crachá com fotografia, usado durante o trabalho de campo. Para garantir a confiabilidade dos dados, a autora do projeto foi responsável pela conferência de todos os formulários recebidos, bem como pela consulta a idosos já entrevistados, os quais foram selecionados por meio de sorteio de $10 \%$ dos formulários recebidos semanalmente, com o intuito de verificar o grau de fidedignidade dos dados. Foi elaborado um manual, utilizado para o treinamento, o qual serviu como fonte de consulta imediata do entrevistador em caso de dúvidas que surgissem durante o trabalho de campo. O manual era constituído de informações sobre a pesquisa, logística do trabalho de campo, material a ser levado para a pesquisa de campo bem como técnicas de coleta dos dados.

Para facilitar a entrada dos entrevistadores em condomínios e edifícios, os mesmos apresentavam, quando necessário, uma carta de solicitação para acesso.

\section{Variáveis de estudo}

As variáveis utilizadas neste estudo foram: sexo, grupo etário (60-69, 70-79 e 80 anos e mais), cor, escolaridade, estado civil e número de residentes no domicílio.

\section{Questões éticas}

Este trabalho foi aprovado pelo Comitê de Ética em Pesquisa (COEP) da Faculdade de Saúde Pública/USP em 07/08/ 2001.

Os idosos receberam explicações a respeito do estudo e, ao concordarem com a 
participação, assinaram o termo de consentimento livre e esclarecido, segundo Resolução n’. 196, de 10 de outubro de 1996 do CONSELHO NACIONAL DE SAÚDE que regulamenta pesquisas envolvendo seres humanos. No caso dos idosos, que por algum motivo, encontravam-se impossibilitados de assinar o termo de consentimento, foi solicitado ao responsável que o fizesse; caso este não estivesse no domicílio, foi realizada nova visita.

\section{Resultados}

Durante a seleção dos domicílios, houve 26 domicílios, cujos idosos não aceitaram participar e os responsáveis por 5 condomínios não autorizaram a entrada dos entrevistadores.

Nos 385 domicílios selecionados para compor a amostra deste estudo, foram entrevistados 483 idosos (68\% do sexo feminino) residentes na cidade de Fortaleza. A média etária dos idosos foi 70,7 anos $(\mathrm{DP}=7,8)$, sendo 70,9 anos $(\mathrm{DP}=7,9)$ para mulheres e 70,3 anos ( $\mathrm{DP}=7,8)$ para homens.

A Tabela 2 apresenta a distribuição dos idosos, segundo sexo e grupo etário. Observa-se que a maior proporção de idosos pertence ao grupo etário 60-69 anos $(49,3 \%)$ e que os idosos com 80 anos e mais, estão presentes em menor proporção (13\%).

Na Tabela 3, é possível observar a caracterização sócio-demográfica dos idosos de Fortaleza. Em relação à cor, os idosos pardos predominam em ambos os sexos, representando $55,1 \%$ do total de idosos estudados, o que também acontece quando se verifica separadamente por sexo. Dos idosos entrevistados, $61,7 \%$ eram analfabetos ou cursaram o primeiro grau incompleto. Os idosos que fizeram graduação ou pós-graduação representam apenas $6,2 \%$ da amostra e o grupo que se apresenta em menor proporção é o de idosos que cursaram ginasial completo/colegial incompleto $(5,8 \%)$. Dos idosos do sexo masculino, $76,9 \%$ eram casados e do sexo feminino, a maior parte era viúva (38,2\%). Em 68,7\% dos domicílios visitados, havia de 2 a 5 residentes.

\section{Discussão}

Este trabalho é parte de um estudo maior que constitui a primeira pesquisa populacional, de base domiciliar, realizada em Fortaleza, que envolve coleta de dados primários relativos a antropometria, consumo alimentar e nível sócio-econômico de idosos. Estudo populacional anterior, realizado por Coelho Filho e Ramos ${ }^{11}$, constituiu o primeiro inquérito domiciliar realizado na cidade, objetivando identificar o perfil multidimensional dos idosos de uma área urbana no Nordeste, com coleta de informações sócio-demográficas, sobre arranjos domiciliares, saúde física, grau de autonomia e utilização de serviços de saúde.

A metodologia utilizada para a execução deste estudo garante a representatividade da população estudada, cujas inferências obtidas poderão ser extrapoladas para a população idosa de Fortaleza. O fato de alguns idosos não aceita-

Tabela 2 - Distribuição dos idosos, segundo sexo e grupo etário. Fortaleza/CE

Table 2 - Distribution of the elderly according to gender and age group. Fortaleza/CE

\begin{tabular}{lcccccc}
\hline & \multicolumn{2}{c}{ Masculino } & \multicolumn{2}{c}{ Sexo } & \multicolumn{3}{c}{ Total } \\
Grupo etário & $\mathrm{n}$ & $\%$ & $\mathrm{n}$ & $\%$ & $\mathrm{n}$ & $\%$ \\
\hline $60-69$ anos & 79 & 50,6 & 159 & 48,6 & 238 & 49,3 \\
$70-79$ anos & 61 & 39,1 & 121 & 37,0 & 182 & 37,7 \\
$\geq 80$ anos & 16 & 10,3 & 47 & 14,4 & 63 & 13,0 \\
Total & 156 & 100 & 327 & 100 & 483 & 100 \\
\hline
\end{tabular}


Tabela 3 - Caracterização sociodemográfica dos idosos residentes em Fortaleza/CE (\%)

Table 3 - Sociodemographic characterization of the elderly residents of Fortaleza/CE (\%)

\begin{tabular}{|c|c|c|c|}
\hline & \multicolumn{3}{|c|}{ SEXO } \\
\hline & Masculino & Feminino & Total \\
\hline \multicolumn{4}{|l|}{ COR } \\
\hline Branco & 41,0 & 46,8 & 44,9 \\
\hline Pardo & 59,0 & 53,2 & 55,1 \\
\hline Amarelo & - & - & - \\
\hline \multicolumn{4}{|l|}{ ESCOLARIDADE } \\
\hline Analfabeto/Primário incompleto & 62,2 & 61,5 & 61,7 \\
\hline Primário completo/Ginasial incompleto & 8,3 & 14,7 & 12,6 \\
\hline Ginasial completo/ Colegial incompleto & 6,4 & 5,5 & 5,8 \\
\hline Colegial completo/ Superior incompleto & 14,1 & 13,4 & 13,7 \\
\hline Superior completo/Pós-graduação & 9,0 & 4,9 & 6,2 \\
\hline \multicolumn{4}{|l|}{ ESTADO CIVIL } \\
\hline Casado & 76,9 & 34,9 & 48,5 \\
\hline Solteiro & 4,5 & 16,5 & 12,6 \\
\hline Viúvo & 12,2 & 38,2 & 29,8 \\
\hline Outros* & 6,4 & 10,4 & 9,1 \\
\hline \multicolumn{4}{|l|}{ RESIDENTES NO DOMICÍLIO } \\
\hline 1 & 5,8 & 8,6 & 7,7 \\
\hline $2-5$ & 69,9 & 68,2 & 68,7 \\
\hline $6 e+$ & 24,3 & 23,2 & 23,6 \\
\hline
\end{tabular}

* Esta classificação se refere a idosos separados, divorciados ou desquitados.

rem participar do estudo e de responsáveis por condomínios não autorizarem a entrada dos entrevistadores, não comprometeu o plano amostral, visto a possibilidade que o mesmo ofereceu de substituição imediata dos idosos que não puderam ou não aceitaram participar da pesquisa.

A população de estudo apresenta em sua maior parte $(67,7 \%)$ mulheres, as quais prevalecem em todos os grupos etários. Esta situação, também encontrada em outros estudos realizados com idosos ${ }^{10-14}$, corrobora os dados nacionais, onde a maioria da população $(50,8 \%)$ é constituída por mulheres $^{8}$. Na cidade de Fortaleza, a situação não é diferente (53,2\% mulheres $)^{6}$.

A predominância de mulheres na população parece ser devida ao aumento da taxa de mortalidade dos homens, bem como à expectativa de vida das mulheres ao nascer, ser maior. De acordo com dados do censo demográfico, realizado em 2000, no Brasil, a esperança de vida das mulheres é superior (72,6 anos) à dos ho- mens (64,8 anos). O mesmo ocorre com a esperança de vida aos 60 anos que das mulheres é 19,4 anos e dos homens é 16 anos. No Ceará, os números são menores, no entanto as mulheres também apresentam esperança de vida ao nascer maior (69,7 anos) que os homens (63,1 anos), assim como a esperança de vida aos 60 anos que para as mulheres é de 18 anos e para os homens é de 15 anos $^{15}$.

A média etária das mulheres deste estudo $(70,9$ anos $(\mathrm{DP}=7,9))$, apesar de similar, foi superior à dos homens (70,3 anos $(\mathrm{DP}=7,8))$. Cabrera e Jacob Filho ${ }^{16}$, em estudo com idosos ambulatoriais, também verificaram média etária similar entre homens e mulheres, sendo a das mulheres superior (72,2 anos e 72,5 anos, respectivamente). Essa situação também é influenciada pelo fato de a esperança de vida das mulheres ser superior à dos homens.

À semelhança da população idosa brasileira e fortalezense ${ }^{8}$, encontrou-se neste estudo, tanto no sexo masculino, quanto 
no feminino maior proporção de idosos pertencentes ao grupo etário 60-69 anos (50,6\% e 48,6\%, respectivamente), caracterizando uma população idosa-jovem. Em estudo realizado com idosos, na cidade de Fortaleza, por Coelho Filho e Ramos ${ }^{11}$, a proporção maior de idosos, também pertencia ao grupo etário 60-69 anos (53\%).

Apesar de no Brasil a maior proporção de idosos ter idade entre 60-69 anos, a proporção de idosos com 80 anos ou mais tem aumentado, assim como em Fortaleza, como mostram os dados dos censos demográficos de 1991 e $2000^{6,17}$, onde a proporção era de $10,9 \%$ e passou para $12,8 \%$, respectivamente. Os idosos com 80 anos ou mais representam $13 \%$ da população deste estudo, onde a população feminina é quase três vezes maior $(74,6 \%)$ que a masculina (25,4\%), confirmando a informação de que as mulheres vivem mais.

A predominância de mulheres na população brasileira e entre os idosos com 80 anos ou mais, assim como neste estudo, pode ser atribuído ao fato de estas idosas, em geral, terem sido menos expostas a determinados fatores de risco, principalmente no trabalho; fumavam e consumiam bebidas alcoólicas em menor quantidade; diferiam dos homens em seu comportamento diante de doenças e incapacidades ${ }^{18}$.

Quanto à cor, a predominância de idosos pardos, verificada neste estudo, pode ser atribuída, principalmente, à grande miscigenação existente no Brasil, por conseguinte em Fortaleza. De acordo com dados do $\mathrm{IBGE}^{8}$, a população de Fortaleza é constituída por $55,1 \%$ de pardos e $2,8 \%$ de pretos. No caso deste estudo, os idosos pretos foram classificados como pardos. Menezes ${ }^{19}$, em estudo realizado com idosos residentes em instituições geriátricas da cidade de Fortaleza, também encontrou maior proporção de idosos pardos $(89,8 \%)$. Assim como neste estudo, no estudo de Menezes ${ }^{19}$, nenhum idoso do grupo amarelo foi abrangido pelo estudo, o que não surpreende, tendo em vista que esse grupo étnico corresponde a apenas $0,17 \%$ dos idosos residentes em Fortaleza ${ }^{20}$.
A informação de estado civil dos idosos deste estudo, onde a proporção maior de homens é casada (76,9 \%) e das mulheres é viúva $(38,2 \%)$ é semelhante aos dados de outros estudos populacionais ${ }^{11,14,21}$. No caso do estudo de Lebrão ${ }^{14}$, realizado com idosos do município de São Paulo, e de Mastroeni ${ }^{21}$, realizado com idosos de Joinville/SC, o número de mulheres viúvas chegou a ser 4 vezes maior que o de homens viúvos. De acordo com Lebrão ${ }^{14}$, isto pode ser atribuído ao fato de as mulheres apresentarem maior esperança de vida e de os homens desse grupo etário casarem mais tarde e terem maior probabilidade de casarem novamente do que as mulheres.

O baixo nível de escolaridade da maioria dos idosos $(61,7 \%$ analfabeto/primário incompleto), confirma o encontrado em outros estudos desenvolvidos com este mesmo grupo etário ${ }^{11,12,21-, 23}$. Considera-se que, possivelmente, essa baixa escolaridade dos idosos, freqüentemente constatada pelos estudos, seja reflexo da taxa de alfabetização nos anos 20-40 do século passado, onde não havia tanta cobrança por nível escolar, como atualmente, e onde o papel das mulheres na sociedade era o de casar, ter filhos e cuidar de casa e, via de regra, não eram estimuladas a estudar.

Quanto ao número de residentes no domicílio, observa-se que a maioria dos idosos $(92,3 \%)$ vive acompanhada e que a maior parte deles $(68,7 \%)$ reside com $2-5$ pessoas. Lebrão ${ }^{14}$ encontrou que $87 \%$ da população idosa do estudo realizado no município de São Paulo vivia acompanhada. Estudos realizados com idosos, que verificaram o tipo de vínculo do idoso com os outros moradores do domicílio, observaram a tendência cada vez maior de idosos morando em domicílios multigeracionais ${ }^{11,12,24}$. Apesar de neste estudo, não haver a informação do tipo de vínculo do idoso com os demais residentes no domicílio, os números confirmam, apenas, a maior proporção de idosos morando acompanhados. Essa situação acontece, possivelmente, não por opção do idoso, mas por limitações financeiras, visto que o idoso, muitas 
vezes é o único suporte financeiro da família, senão o mais expressivo.

A proporção de idosos que viviam sozinhos foi $7,7 \%$, número similar ao encontrado por Coelho Filho e Ramos ${ }^{11}$ (6,3\%) em estudo populacional realizado em Fortaleza. Ramos et al. ${ }^{12}$, em pesquisa realizada com idosos residentes no município de São Paulo, encontraram proporção maior (10\%) de idosos morando sozinhos, assim como Lebrão ${ }^{14}(13,1 \%)$. Essa proporção maior de idosos morando sozinhos, encontrada nos estudos de Ramos et al. ${ }^{12} \mathrm{e}$ de Lebrão ${ }^{14}$, parece refletir uma situação de maior independência financeira e físi- ca desses idosos, visto que viver em São Paulo, região sabidamente mais desenvolvida que Fortaleza, pode proporcionar maiores oportunidades econômicas e de serviços de saúde.

Como visto, em geral os resultados aqui apresentados confirmam estudos anteriores, os quais mostram a heterogeneidade da população idosa. Diante disso, observa-se a importância do planejamento de políticas públicas direcionadas às necessidades específicas da população idosa, além de outros estudos que possibilitem ampliar o conhecimento a respeito dessa população.

\section{Referências}

1. Kalache A, Veras RP, Ramos LR. O envelhecimento da população mundial: um desafio novo. Rev Saúde Pública 1987; 21(3): 200-10.

2. Ramos LR, Veras RP, Kalache A. Envelhecimento populacional: uma realidade brasileira. Rev Saúde Pública 1987; 21(3):211-21.

3. Carvalho JAM, Garcia RA. O envelhecimento da população brasileira: um enfoque demográfico. Cad Saúde Pública 2003; 19(3): 725-33.

4. Breuer B, Wallenstein S, Feinberg S, Camargo MJF, Libow LS. Assessing life expectancies of older nursing home residents. JAm Geriatr Soc 1998; 46: 954-62.

5. Ramos LR. O país do futuro não pensa no futuro. Gerontologia 1995; 3(1): 52-4.

6. Fundação IBGE. Censo Demográfico 2000: Resultado do universo - Fortaleza. 2000a.

7. Fundação IBGE. Censo Demográfico 1980: características da população e dos domicílios - Resultado do universo. Rio de Janeiro, 1980.

8. Fundação IBGE. Censo Demográfico 2000: características da população e dos domicílios - Resultado do universo. Rio de Janeiro, p. 1-520, 2000b.

9. Fundação IBGE. Pesquisa Nacional por amostra de domicílios/PNAD-Manual de listagem. p. 1-82, 2002.

10. Menezes TN, Marruci MFN. Antropometria de idosos residentes em instituições geriátricas, Fortaleza, CE. Rev Saúde Pública 2005; 39(2): 169-75.

11. Coelho Filho JM, Ramos LR. Epidemiologia do envelhecimento no Nordeste do Brasil: resultados de inquérito domiciliar. Rev Saúde Pública 1999; 33 (5): 44553.
12. Ramos LR, Rosa TEC, Oliveira ZM, Medina MCG, Santos FRG. Perfil do idoso em área metropolitana na região sudeste do Brasil: resultados de inquérito domiciliar. Rev Saúde Pública 1993; 27(2): 87-94.

13. Tavares EL, Anjos LA. Perfil antropométrico da população idosa brasileira. Resultados da Pesquisa Nacional sobre Saúde e Nutrição. Cad Saúde Pública 1999; 15 (4): 327-33.

14. Lebrão ML. O projeto SABE em São Paulo: uma visão panorâmica. In: Lebrão ML, Duarte YAO. SABE - Saúde, Bem-estar e Envelhecimento - O projeto SABE no Município de São Paulo: uma abordagem inicial. Brasília: Organização Pan-Americana de Saúde; 2003. p. 35-43.

15. Fundação SEADE. Anuário estatístico de saúde do Brasil. Brasília, 2002.

16. Cabrera MAS, Jacob Filho W. Obesidade em idosos: prevalência, distribuição e associação com hábitos e comorbidades. Arq Bras Endocrinol Metab 2001; 45(5): 494501.

17. Fundação IBGE. Censo Demográfico 1991: características da população e dos domicílios - Resultado do universo. Rio de Janeiro, 1991.

18. Veras RP. País jovem com cabelos brancos: a saúde do idoso no Brasil. Rio de Janeiro: Editora Relume-Dumará/ EDUERJ; 1994.

19. Menezes TN. Avaliação antropométrica e do consumo alimentar de idosos residentes em instituições geriátricas da cidade de Fortaleza/Ceará [dissertação de Mestrado]. São Paulo: Faculdade de Saúde Pública da USP; 2000.

20. Fundação IBGE. Censo Demográfico 2000: Resultados da amostra - Ceará (municípios). 2000c. 
21. Mastroeni MF. Estado nutricional e consumo de macronutrientes de idosos da cidade de Joinville, SC [Tese de Doutorado]. São Paulo: Faculdade de Saúde Pública da USP; 2004.

22. Lima-Costa MFF, Uchoa E, Guerra HL, Firmo JOA, Vidigal PG, Barreto SM. The Bambuí health and ageing study (BHAS): methodological approach and preliminary results of a population-based cohort study of the elderly in Brazil. Rev Saúde Pública 2000; 34(2): 126-35.
23. Zaitune MPA, Barros MBA, César CLG, Carandina L, Goldbaum M. Hipertensão arterial em idosos: prevalência, fatores associados e práticas de controle no município de Campinas, São Paulo, Brasil. Cad Saúde Pública 2006; 22(2): 285-294.

24. Ramos LR, Toniolo JN, Cendoroglo MS, Garcia JT, Najas MS, Perracini M et al. Two-year follow-up study of elderly residents in S. Paulo, Brazil: methodology and preliminary results. Rev Saúde Pública 1998; 32 (5): 397 407.

Recebido em: 30/05/06 Versão final reapresentada em: 26/02/07

Aprovado em: 13/03/07 